\title{
LE PRINTEMPS ARABE ET LA PÉRENNITÉ DES DROITS DE LA FEMME
}

\author{
THE ARAB SPRING AND THE SUSTAINABILITY OF WOMEN'S RIGHTS \\ ABSTRACT
}

Fatima Roumate ${ }^{1}$

\section{RESUME}

Deux ans après, les conséquences du printemps arabe sont une masculinité des pouvoirs et un recul en ce qui concerne les droits des femmes qui ont payé cher le prix du changement. Subséquemment, les islamistes venant aux pouvoir après les révolutions arabes parlent d'une démocratisation mais sans les droits des femmes. Une démocratisation qui favorise l'autorité parentale et qui touche profondément la dignité de l'autre moitié de la société. Cela met sur table la question de la sécurité voir même la sécurité juridique.

La pérennité des droits des femmes représente un risque pour la sécurité et une menace aux acquis réalisés par les associations féminines après de langues années de militantismes. Elle a un cout et un impact négatif sur le développement du monde arabe.

En effet, cette pérennité met en cause aussi la légitimité de ces gouvernants choisis par des hommes et des femmes et qui sont inaptes de protéger les droits de la moitié de la société qui les a choisis et acceptés. Cela, tout en considérant que le contrat social n'est que le choix et l'acceptation des gouvernants qui doivent, en contre partie, assurer la sécurité et protéger les droits humains des gouvernés hommes et femmes.

\section{Mots clès}

printemps arabe, droits, sécurité, femmes

\section{ABSTRACT}

Two years later, the consequences of the Arab Spring are a masculinity of powers and a retreat regarding the rights of women who paid a high price for the change. Subsequently, the Islamists come to power after the Arab revolutions that talk about democratization, but without the rights of women. The democratization that promotes the parental rights and affects profoundly the dignity of the other half of society. This brings to the table the issue of safety or even legal certainty.

The sustainability of women's rights is a risk to safety and a threat to the achievements of women's groups after years of militancy language. It has a cost and a negative impact on the development of the Arab world.

Indeed, sustainability also implies the legitimacy of governors chosen by men and women who are not able to protect the rights of half of society who has chosen and accepted them. This considering that the social contract is only the selection and acceptance of leaders who must, in return, ensure the safety and protect the human rights of governed men and women.

\section{Keywords}

Arab Spring, rights, safety, women

Professeur à l'Université Mohammed V Agdal, Rabat Faculté des Sciences Juridiques, Economiques et Sociales. Présidente de l'Institut International de la Recherche Scientifique. 
Deux ans après, les conséquences du printemps arabe est un recul en ce qui concerne les droits de la femme qui ont payé cher le prix du changement et une masculinité des pouvoirs. Ainsi dans plusieurs pays arabes la féminisation des instances de décision est à l'ordre du jour. Cela met sur table la question de la sécurité voir même la sécurité juridique en tant que principe de droit public. Les islamistes venant aux pouvoir après les révolutions arabes parlent d'une démocratisation mais sans les droits de la femme. Une démocratisation qui favorise l'autorité parentale et qui touche profondément la dignité de l'autre moitié de la société. Pourtant la démocratie en tant que notion n'a pas de sens sans les droits des femmes.

Il est donc légitime de se poser la question sur la sécurité et le processus démocratique dans le monde arabe en considérant les menaces aux droits de la femme après le printemps arabe.

D'après cette publication, nous allons mettre le point sur l'état des lieux des droits de la femme après le printemps arabe, la question de la sécurité et le processus démocratique inconcevable sans la reconnaissance et le respect des droits de l'autre moitié de la société.

\section{LE PRINTEMPS ARABE : LES DROITS DE LA FEMME SONT MENACÉS}

La plupart des révolutions qui ont marqué le monde arabe en 2011 ont été initiées par des femmes. Au Caire, Asmaa Mahfouz a appelé à se protester contre le Régime Moubarak le 18 janvier 2011.

If we have honour and want to live in dignity in this country, we must all go down to Tahrir Square on 25 January... We must demand our fundamental human rights as human beings...I will go to Tahrir Square. I will say no to corruption! I will say no to this system!". Asmaa Mahfouz, video blog, 18 January.
Au Yemen, c'est Twakkol Karman qui a appelé les manifestants à s'élever contre le régime corrompus.

Women are no longer victims, they have become leaders, they are at the forefront of demonstrations... The participation of young people, men and women, without any ideological or political background, has made this movement explode"' Tawakkul Karman, activist, Nobel Peace Prize Laureate, FIDH Press Conference, 7 November 2011.

En Libye aussi se sont les femmes qui sont à l'origine de la révolte. En Tunisie les femmes ont été présentes et ont joué un rôle important dans le changement du régime.

Au Maroc, le mouvement féminin a contribué largement à la transition démocratique et au changement qui a mené à la mise en place d'une nouvelle constitution.

Pourtant l'histoire confirme encore une fois que les droits de la femme sont la première chose à écarter par les hommes qui monopolisent les instances de prise de décision. Cela confirme la thèse de Simone de Beauvoir "La majorité des femmes a toujours été tenue à l'écart de la marche du monde, parce que les hommes, qui se posèrent d'emblée comme les seuls responsables, leur refusèrent les possibilités d'une existence autonome. ${ }^{4} »$. Pour Simone de Beauvoir, la domination des femmes par les hommes ne s'explique pas par les différences, physique, morphologique et sexuel et elles ne sont pas fondées en nature mais elles sont dues à une situation de fait établie depuis l'origine des temps. Les hommes ont assimilé la femme à son corps, à son sexe et ils l'ont renfermé dans les rôles de mères et de servantes. Ceci dit, la fonction reproductrice ne suffit pas pour expliquer la domination de la femme par l'homme car ce sont les hommes qui ont pensé cette situation comme secondaire et qui l'ont confirmé dans les institutions et les lois 5 .

\footnotetext{
Fédération internationale des ligues des droits de l'Homme, Monde arabe : quel printemps pour les femmes, document électronique, www.fidh.org 2012, p. 16.

3 Ibid, p. 40.

4 Daniel Armogathe, Le deuxième sexe Beauvoir, Hatier, Paris 1977, p. 6.

Ibid pp. 13-14.
} 
Cela explique le résultat négatif de la contribution massive des femmes aux changements dans le monde arabe qui était $2 \%$ seulement des femmes au parlement en Egypte et " aucune femme n'a fait partie du Comité constitutionnel, ni du Comité civil de consultation, dont le nom, "Conseil des hommes sages ", est en soi discriminatoire ${ }^{6}$ ». En 2010, la participation politique des femmes en Egypte était $12 \%$ contre uniquement $2 \%$ en 2011 après l'abrogation de la loi mise en place sous la présidence de Moubarak et qui a instauré un quota de 64 de siège réservés aux femmes ${ }^{7}$.

Au Maroc, une seule femme au gouvernement islamiste et 30 hommes, cela après une nouvelle constitution caractérisée par plusieurs nouveautés, notamment le principe de la parité et une autorité pour la parité et la lutte contre toutes les formes de discrimination (l'article 19 de la constitution de 2011).

Pourtant, force est de signaler une contradiction et une fracture indéniables entre la nouvelle Constitution qui a consacrée le principe de la parité et la composition du gouvernement islamiste contenant une femme et 30 hommes ministres ce qui constitue une violation grave de la Constitution. En effet ce recul ne se traduit pas uniquement par le déclin de la représentation politique des femmes dans le Gouvernement qui est passé de sept ministres en 2007 à un poste ministériel dans l'Exécutif actuel, mais se traduit aussi par la qualité du portefeuille consacré à la femme qui réduit ses capacités à un rôle stéréotypé. Le seul portefeuille accordé à la femme par le nouveau gouvernement marocain prouve le champ étriqué consacré aux droits des femmes qui réduit ces questions à la famille et à l'enfance. Alors que les droits des femmes est une affaire qui doit être prise en considération et d'une manière transversale dans la mise en place de toutes les politiques publiques.

Le repli au niveau des droits des femmes se traduit aussi avec le gouvernement islamiste par un discours qui touche profondément la dignité des femmes et freine tous les efforts du mouvement féminin qui s'est investit depuis de longues années pour assurer la justice sociale, l'égalité entre tous les citoyens et l'intégration de tous et de toutes dans le processus démocratique et de développement sans aucune discrimination fondée sur le genre.

En Tunisie, le pays qui a été pionnier dans la région en ce qui concerne l'égalité des sexes ${ }^{8}$, le gouvernement islamiste tunisien ne contient que 3 femmes dans 41 ministres alors que le dernier gouvernement de Ben Ali contenait 4 femmes ministres. Pour la participation des femmes à la Chambre des députés à l'époque de Ben Ali était $30 \%$ et uniquement $27,2 \%$ de femmes élues à l'Assemblée constituante ${ }^{9}$.

Cette détérioration au niveau des droits des femmes et des acquis des associations féminines dans le monde arabe et qui a été l'un des résultats frappant du printemps arabe nous incite à poser la question sur la sécurité des droits des femmes arabes.

\section{QUELLE SÉCURITÉ POUR LES DROITS DES FEMMES APRÈS LE PRINTEMPS ARABE?}

Le premier devoir de l'Etat est sans doute le maintien de la paix entre les citoyens et les citoyennes. La sécurité au niveau interne ne se limite pas uniquement au maintien de l'ordre public. Cette notion a évolué avec l'évolution des sociétés. Ainsi, la sécurité physique des personnes et des biens, la sécurité sociale, la sécurité sanitaire, la protection du

6 Fédération internationale des ligues des droits de l'Homme, Monde arabe : quel printemps pour les femmes, document électronique, www.fidh.org

2012 , p. 5

7 Ibid, p. 23

8 Conseil de l'Europe, Egalité entre les femmes et les hommes : une condition du succès du printemps arabe, Assemblée Parlementaire, 05 avril 2012, Document electronique, http://assembly.coe.int

9 Fédération internationale des ligues des droits de l'Homme, Monde arabe : quel printemps pour les femmes, document électronique, www.fidh.org 2012, p. 12-13. 
consommateur, celle de l'environnement, etc, ne concerne pas uniquement les hommes. Les femmes aussi doivent bénéficier de la sécurité. Le devoir de l'Etat et du gouvernement est d'assurer la sécurité des femmes à tous les niveaux tout simplement car elles constituent l'autre moitié qui a choisi et qui a accepté les gouvernants. Le concept de la sécurité humaine reste spécialement pertinent pour les pays arabes qui ont limité ce concept au contexte international et au domaine militaire et en contre partie ils ont marginalisé la sécurité humaine qui s'étend à tous les domaines de la vie active des gens ${ }^{10}$.

Peut - on dire que les gouverants venant au pouvoir après les révolutions arabes sont aptes à garantir la sécurité (physique, des biens, la sécurité sociale, la sécurité sanitaire,...) à l'autre moitié de la société? Cela, en considérant que les femmes durant les révolutions arabes et après ont subi toute forme de violence : viols, test de virginité, kidnapping.

En fait cet état d'insécurité physique n'est que le prolongement des discriminations que ces pays vivent dans les temps de relative normalité comme le confirme la résolution du conseil de sécurité $1325^{11}$.

En Egypte les testes de virginité est une violence physique et morale aux femmes et à leurs familles. Le gouvernement Egyptien n'a pas garantie la sécurité à ces femmes qui ont endurées toutes sortes de violence par l'armée le lendemain de départ de Moubarrak. Durant les révolutions qui ont accompagnées le transfert de pouvoir en Egypte, les manifestantes ont été menacées, harcelées et agressées sexuellement. Le cas de Caroline Sinz qui a été agressée sexuellement par un groupe d'hommes et la journaliste Lara Logan qui a été agressée sexuellement par 40 hommes à Tahrir ${ }^{12}$. Larmée et la police ont aussi exercé toutes sortes de violence sur les femmes le lendemain de la journée mondiale des femmes (le 9 mars 2011). L'exemple de Samira Ibrahim qui a été forcé de faire un test de virginité par l'armée. Plusieurs membres de l'armée y compris le Major Général Abdel Fattah Al Sisi, et le Chef de renseignement militaire de l'Egypte ont admit cette pratique ${ }^{13}$. Alors comment peut-on dire que le principal devoir des gouvernants est de garantir la sécurité des gens quand eux même menacent cette sécurité ?

En Libye, plusieurs femmes ont été violé par l'armée de Kaddafi est ont été tuées par la suite par les membres de leurs familles pour " laver » le déshonneur. Plusieurs pères, maries et frères ont tuées leurs filles, leurs épouses et leurs sœurs pour éviter qu'elles soient violées par l'armée. Le cas d'Iman Al-Obeidi la journaliste violée par la force de sécurité ${ }^{14}$.

$\mathrm{Au}$ Yemen, les femmes qui ont contribué aux manifestations du 10 et 17 octobre et du 19 avril 2011 ont subi toute sorte de violence de la part des forces de la sécurité ${ }^{15}$.

$\mathrm{Au}$ Maroc, et selon le Haut Commissariat au Plan « plus de $15 \%$ des femmes ont déclaré avoir subi une violence physique. Ce taux est 2 fois plus élevé dans les villes que dans les campagnes $(19,4 \%$, contre $9 \%) »^{16}$.

\footnotetext{
10 Paula Banerjee, Pirkko Poutiainen, Ishita Dey, Shiva Dunghana, Wanza Kiolo, Päivi Matila et Martin Muhindi, Women, Peace and Security, Implementation of UN Security Council Resolution 1325 in the context of Finnish Development Policy with case studies from Kenya, Nepal and North-Est India, Finnish Ministry for Foreign Affairs, Helsinki, 2010, p. 34

11 Françoise Nduwimana, La Résolution 1325 du Conseil de sécurité de l'ONU sur les femmes, la paix et la sécurité Comprendre les implications, remplir les obligations, Bureau de la Conseillère spéciale pour la parité entre les sexes et la promotion de la femme OSAGI, Département des Affaires économiques et sociales (DAES), Commission économique des Nations Unies pour l'Afrique (CEA) New York, p. 8. http://www.un.org/womenwatch/osagi/cdrom/documents/Background_Paper_Africa_fr.pdf

12 Fédération internationale des ligues des droits de l'Homme, Monde arabe : quel printemps pour les femmes, document électronique, www.fidh.org 2012, p. 18 .

13 Ibid

14 Ibidem p. 28

15 Ibid, p. 40.

16 Haut Commissariat au Plan, Enquête nationale sur la prévalence de la violence à l'égard des femmes au Maroc, 2009, document d'internet site web : www.hcp.ma, p. 32.
} 
Alors quelle sécurité les gouvernants arabes peuvent garantir aux femmes? sont-ils vraiment aptes à assurer cette condition principale du contrat social à l'autre moitié de la société?

Sans la satisfaction des besoins de la moitié de la société, sans la garantie d'une sécurité voir même une sécurité juridique aux droits des femmes déjà acquis, la légitimité des gouvernants venant au pouvoir après les révolutions arabes de 2011 est mise en question.

La sécurité juridique est un principe de droit public, le contrat social n'est fondamentalement que l'échange d'une liberté contre la sécurité ${ }^{17}$.

La sécurité juridique est parmi les obligations de l'Etat. Selon Jean-Pierre PUISSOCHET et Hubert LEGAL «la sécurité juridique appartient à une galaxie bien autrement diverse, puisqu'on $y$ trouve des impératifs occasionnellement discordants et qu'en relèvent à la fois le respect de la légalité et les atténuations qu'il doit recevoir en vue de la protection de droits acquis ou légitimement attendus» ${ }^{18}$. Ce principe vise à assurer la stabilité des situations juridiques établies et le respect des droits acquis ${ }^{19}$.

Cette sécurité doit être préservée d'une manière transversale dans toutes les politiques publiques. Ce qui n'a pas été respecté par les gouvernants venant au pouvoir après les révolutions arabes.

Le déclin au niveau des droits des femmes après le printemps arabes constitue un risque pour cette sécurité juridique et une menace aux acquis juridiques réalisés par les associations féminines après de langues années de militantismes.
Les exemples dans ce sens sont très nombreux. En Egypte, plusieurs réformes juridiques ont eu lieu entre 2000 et 2010, notamment la criminalisation de la mutilation génitale féminine, la garde des enfants, le divorce et l'âge de mariage. Néanmoins le lendemain de la démission de Moubarak les islamistes ont dénoncé ces réformes et ont demandé l'abrogation de toutes les lois qui sont incompatibles avec la charia ${ }^{20}$.

En Tunisie, la convention CEDAW a été ratifié en 1985 mais en gardant plusieurs réserves. En 2011, le gouvernement de transition a déclaré que les réserves seraient levées mais aussi l'article 1er de la constitution selon lequel la constitution prime sur toutes les conventions internationales a été maintenu ${ }^{21}$.

En Libye, la première chose que le chef du gouvernement par intérim, Moustafa Abdel Jalil, promit de faire fut de lever les restrictions concernant la polygamie.

Au Maroc le vote d'une nouvelle réforme du code de la famille (le projet de loi 70-03) qui vise la réforme de l'article 20 et 21 concernant l'âge de mariage des filles à 16 ans au lieu de 18 ans par la chambre des conseillers constitue un exemple concret du recul aux niveau des droits acquis et pour lequel le mouvement féminin a milité pendant de longues années.

Ces différentes menaces au principe de la sécurité juridique qui vise la préservation des droits déjà acquis ne mettent pas en cause la légitimité de ces gouvernants qui ont choisi de mettre en place une démocratie qui traduit leur pensée masculine? La masculinité des pouvoirs et des postes de décision après les révolutions arabes n'est pas une atteinte au principe de la sécurité juridique?

\footnotetext{
17 Didier Truchet, Le droit public, PUF, Paris, 2010, p. 61.

18 Jean-Pierre PUISSOCHET, Hubert LEGAL, Le principe de sécurité juridique dans la jurisprudence de la Cour de justice des Communautés européennes, Cahiers du Conseil constitutionnel $n^{\circ} 11$ (Dossier : Le principe de sécurité juridique), décembre 2001 p. 7, http://www.conseil-constitutionnel.fr/conseil-constitutionnel/francais/cahiers-du conseil/cahier-n-11/leprincipe-de-securite-juridique-dans-la-jurisprudence-de-la-cour-de-justice-des-communautes-europeennes.52123.html

19 Didier Truchet, opcit, p. 62.

20 Fédération internationale des ligues des droits de l'Homme, Monde arabe : quel printemps pour les femmes, document électronique, www.fidh.org 2012, p. 24-25.

21 Ibid p. 12.
} 
La violation de l'esprit de l'article 19 de la nouvelle constitution par le gouvernement conservateur marocain en accordant un seul portefeuille à la femme ne touche t-il pas sa légitimité juridique? Le non respect du principe de la parité contenu dans la loi suprême du pays ne représente- t-il pas une atteinte à la légalité?

Dans ce sens, il est impératif de signaler qu'il $\mathrm{n}$ y a aucune femme présidente d'une université au Maroc, au moment ou les 15 universités existantes sont présidées par des hommes. Aucune femme ne préside une chambre de commerce, et on signale l'absence de statistiques sur le nombre de femmes qui président les entreprises publiques ou qui font partie du conseil d'administration. De plus le gouvernement marocain actuel n'a pas mis en place aucune mesure qui peut réparer ce déséquilibre voir même cette discrimination fondée sur le genre. Les parties politiques qui forment le gouvernement actuel n'utilisent la carte de la lutte contre la discrimination fondée sur le genre que pendant leurs compagnes électorales. Ils ne pensent à l'autre moitié de la société que pour garantir le maximum possible des voix tout en omettant que le jeu électoral est en fait un mécanisme par lequel le contrat social est établi et que la non satisfaction des besoins du peuple (hommes et femmes) conditionne la légitimité du pouvoir.

La notion de légitimité en tant que base pour l'analyse du phénomène du pouvoir a évolué dans les temps modernes afin qu'il reflète le choix des gouvernants dans un système politique et l'acceptation de ce choix par les gouvernés, hommes et femmes.

Cette légitimité est mise en cause en considérant l'absence des mesures juridiques, institutionnelles et financières qui peuvent garantir la parité homme-femme. Dans ce sens, il est impératif de signaler que le développement est inconcevable sans la contribution des femmes à l'identification, la réalisation et l'évaluation des politiques publiques et sans la considération de l'approche genre et l'approche basée sur les droits dans le processus de développement.

Un système politique qui ne prend pas en considération les besoins des citoyens et des citoyennes, et qui ne préserve pas les droits de l'homme et les droits des femmes n'a pas de légitimité.

Tout serait perdu, si les pouvoirs sont masculinisés et l'Etat de droit ne serait existé en mettant tous les pouvoirs uniquement entre les mains des hommes et sans la participation de l'autre moitié de la société. Une autre sorte de tyrannie continuera à exister tant que les hommes monopolisent les postes de décision dans le monde arabe.

Linsécurité des droits des femmes nous mène à repenser cette relation très étroite entre la sécurité humaine et le développement humain confirmée par le rapport du PNUD de $2013^{22}$. En fait, Karim Hussein, Donata Gnisci et Julia Wanjiru affirment que la communauté internationale a commencé à mettre en relation les questions de sécurité avec celles de développement au début des années $90^{23}$. Selon le PNUD, la sécurité humaine est considérée comme le pilier du développement humain ${ }^{24}$.

Subséquemment, le coût de la non intégration de l'autre moitié de la société dans le développement du monde arabe est très cher. Cette marginalisation du rôle de la femme peut porter préjudice à toutes les stratégies de bonne gouvernance tout en considérant que le but ultime de cette dernière n'est autre que l'amélioration de la qualité de vie des personnes.

\footnotetext{
22 Programme des Nations Unies de Développement, L'essor du Sud, le progrès humain dans un monde diversifié, Rapport sur le développement humain 2013, p. 40 http://www.undp.org/content/dam/undp/library/corporate/HDR/2013GlobalHDR/French/HDR2013\%20Report\%20French.pdf

23 Karim Hussein, Donata Gnisci et Julia Wanjiru, Sécurité et sécurité humaine : Présentation des concepts et des initiatives, Quelle conséquences pour l'Afrique de l'Est, OCDE, 2004, p. 12. http://www.oecd.org/fr/csao/publications/38826711.pdf

24 PNUD, Les defis de la securite humaine dans les pays, Rapport sur le développement humain dans le monde Arabe 2009 , p 22. http://www.arab-hdr.org/publications/other/ahdr/ahdr2009f.pdf
} 


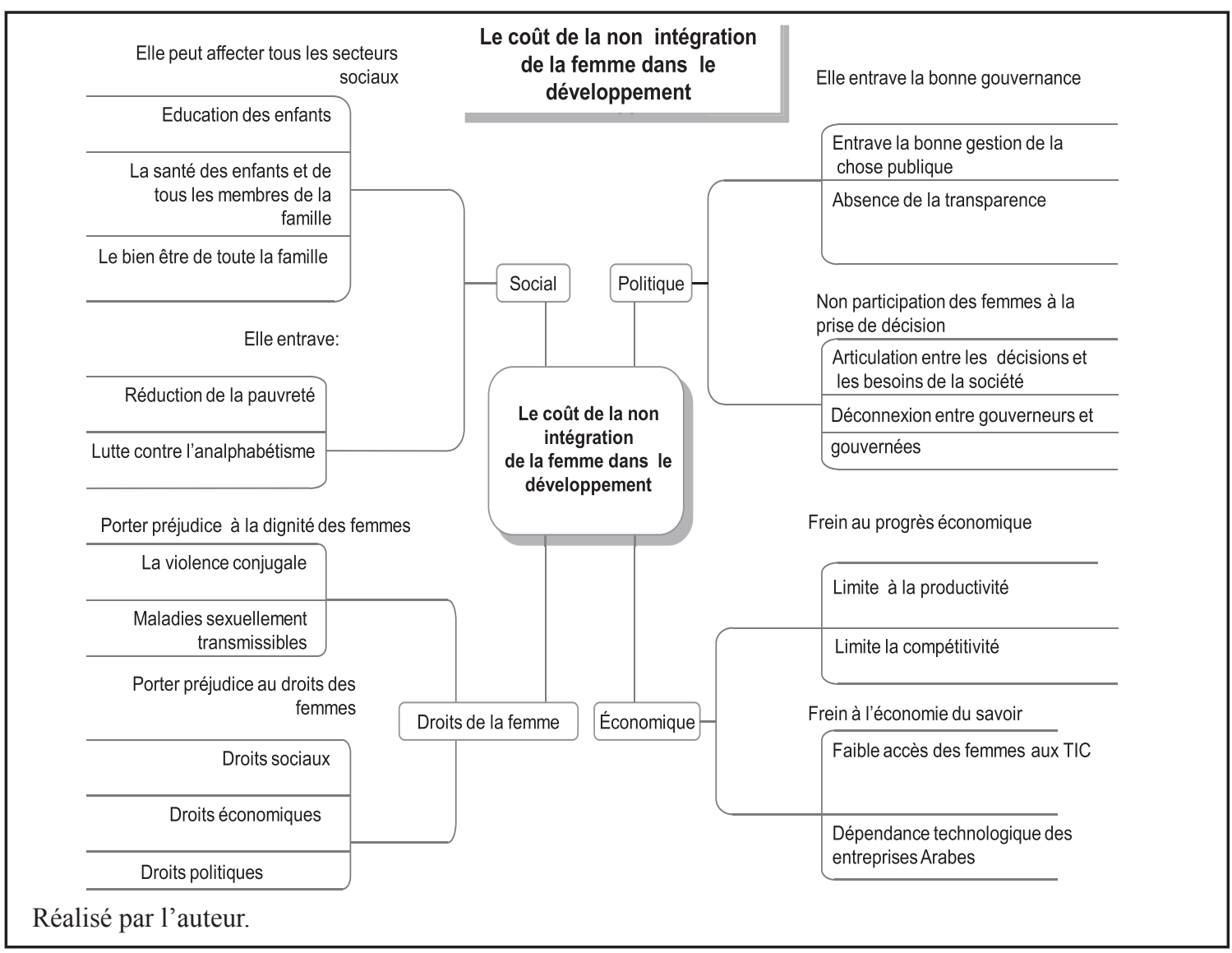

Dans ce sens, il est impératif de rappeler que les politiques du développement des pays arabes ont été fondées depuis les années cinquante et même au début des années quatre vingt dix sur la mise en place des structures générales ayant comme objectif principal la réalisation de la croissance sans prendre en considération les structures économiques et sociales. Autrement dit, les politiques du développement des pays arabes ont été fondées sur un cadre théorique qui a traité la relation entre la femme et le développement mais d'un point de vue masculin visant la satisfaction des besoins fondamentaux de la femme en tant que mère, femme de foyer et ressource humaine qui contribue largement à la croissance mais sans considérer l'amélioration de ces conditions économiques et sociales en tant qu'individu.

Le développement fondé sur ce cadre théorique suppose que l'Etat peut garantir les besoins effectifs des femmes et que cela constitue une priorité pour les décideurs. Pourtant cette hypothèse est encore loin d'être réelle car les décideurs dans le monde arabe, qui sont des hommes, n'ont jamais considéré que les besoins des femmes en tant qu'individu est une priorité. Cela s'explique par l'absence de l'approche genre dans le partage des rôles entre hommes et femmes dans la prise de décision. En fait, la femme dans le monde arabe ne contribue pas à la prise de décision dans tous les niveaux, commençant par son foyer et jusqu'aux plus hautes institutions étatiques. Dans certains pays arabes, les décisions concernant la famille telle que le choix de l'école pour les enfants ou même l'utilisation des revenus de la femme elle-même sont monopolisées par l'homme.

Dans le même sillage, ce cadre théorique considère que les femmes peuvent avoir accès à leurs besoins dès que les gouvernements sont aptes à les garantir et les mettre à disposition des femmes. Cependant on constate dans certains pays arabes que même si les gouvernements assurent les besoins fondamentaux aux femmes, ces dernières ne peuvent pas en bénéficier sans l'autorisation des hommes (Père, frère, mari et même fils). En conséquence, la femme reste une ressource 
humaine qui contribue à la croissance mais pas au développement puisqu'elle n'est pas intégrée dans ce développement, en tant que bénéficiaire mais encore plus en tant que décideur.

A l'aube de la mondialisation voir même la mondialisation technologique et l'émergence des sociétés et des économies du savoir dont l'élément moteur est les ressources humaines, la non intégration de la femme dans les politiques du développement représente aussi un frein à l'émergence des sociétés arabes vers une société du savoir et un obstacle à la sécurité humaine.

Comment peut- on assurer l'émergence vers une société du savoir avec la marginalisation de la moitié des ressources humaines? $\mathrm{Au}$ moment ou le monde arabe a besoin de doubler ces efforts de Recherche et Développement pour répondre aux enjeux imposés par la mondialisation technologique on constate aussi la monté de la discrimination au niveau de l'accès des femmes à l'emploi qualifié et aux postes de dirigeantes dans le secteur public et privé. La preuve est ces différents discours des gouvernants islamistes qui confirment qu'ils sont pour le retour des femmes à leurs foyers.

La non intégration de la femme dans le développement signifie la non mobilisation au profit de toute la société arabe des énergies et des compétences féminines qui représentent $50 \%$ des ressources humaines disponibles.

Elle signifie aussi la non réalisation des objectifs du millénaire notamment. En conséquence, le monde arabe continue à perdre des points au niveau des différents classements internationaux du développement humain et démocratique au lieu d'améliore sa situation.

La non intégration de la femme dans le développement $\mathrm{du}$ monde arabe et les atteintes à leurs droits nous incite à se poser la question sur la démocratie. Comment peut- on concevoir une démocratie sans les droits des femmes?

\section{LE PRINTEMPS ARABE: UNE DÉMOCRATISATION SANS LES DROITS DES FEMMES}

Pour Montesquieu, «lorsque dans la république, le peuple en corps a la souveraine puissance, c'est une démocratie» ${ }^{25}$ et selon, le président Lincoln la démocratie est «le gouvernement $\mathrm{du}$ peuple, par le peuple, pour le peuple» ${ }^{26}$. La plupart des politologues ont confirmé cette définition. Pourtant la réalité dans les pays arabes nous dit que le gouvernement du peuple se fait uniquement par les hommes.

La démocratie est inconcevable sans la parité et sans la protection des droits des hommes et des femmes. Cela est le principal objectif du mouvement féminin au Maroc. Ainsi, plusieurs réformes législatives et institutionnelles ont vu le jour grâce aux efforts de ce mouvement, en l'occurrence, les nouvelles dispositions de la constitution concernant spécialement la parité. Quoique, et au-delà du texte de la constitution, les obstacles à la parité, aux droits des femmes et à la démocratie demeurent dans l'application de ce texte. La composition du nouveau gouvernement est une preuve entre autres.

Dans ce cadre, le déclin au niveau des droits des femmes après le printemps arabe met à l'ordre du jour le débat sur la compatibilité entre l'islamisme et la démocratie.

Une première lecture des différents discours des nouveaux gouvernements islamistes confirme l'importance donnée à cette question. Ainsi, d'près la lecture du programme du nouveau gouvernement marocain; la démocratie est au centre des préoccupations du chef du gouvernement islamiste. Néanmoins la question qui se pose est quel est la conception de la démocratie adoptée par le parti islamiste qui préside le gouvernement actuel au Maroc? Autrement dit, si on considère que

\footnotetext{
${ }_{25}$ Francis Dupuis-Déri, Qu'est-ce que la démocratie?, Horizons philosophiques, vol. 5, n 1, 1994, p. 84-95, http://id.erudit. org/iderudit/800967ar, p. 88.

26 Ibid.
} 
le maintien de la continuité des efforts menés par les anciens gouvernements au Maroc ne peut que favoriser la voie de la démocratie, il faut rappelé qu'en fait la démocratie n'est pas un choix pour les partis islamistes venant au pouvoir au Maroc comme dans d'autres pays arabes mais c'est une obligation politique imposée par les enjeux internes qui s'inscrivent dans le cadre de la réalisation des objectifs du millénaire et les enjeux externes imposés par la mondialisation et par la crise économique actuelle.

Le respect du principe de la continuité qui luimême garanti la sécurité voir même la sécurité juridique, notamment les acquis juridiques concernant la situation de la femme qui est au centre de débat concernant l'arrivée des islamistes au pouvoir. Ces derniers ont été toujours pour le retour des femmes au foyer comme élément fondamental du développement et de la consolidation de la famille. La continuité dans la voie de la démocratie par les islamistes au Maroc a été signalé d'après le programme gouvernemental présenté par le chef du gouvernement devant le parlement le 29 Janvier $2012^{27}$ comme elle a été aussi remarquée dans les discours du Ministre des affaires générales et de la gouvernance lors de la réunion de Davos qui a affirmé qu'il n'y a " aucune contradiction entre démocratie et Islam ».

Toutefois, comment peut - on croire qu'il $\mathrm{n}$ y a aucune contradiction entre la démocratie et la pensée des islamistes qui sont au pouvoir dans le monde arabe au moment ou les inégalités homme-femme sont encore plus flagrantes. Selon le rapport mondial sur l'inégalité entre les sexes du Forum Economique Mondiale de 2012, les pays arabes sont classés parmi les derniers. Ainsi le Maroc dont le gouvernement se félicite chaque année par les progrès à ce niveau d'après son rapport sur le budget genre occupe la place 129ème, l'Egypte 126ème et le Yemen $135^{\text {ème28 }}$.

De quelle démocratie parle - $\mathrm{t}$ - on dans un pays qui contient une loi qui autorise la violence physique faite aux femmes comme le confirme Mona Eltawahy "Quand un article du code pénal dit que si une femme a été battue par son mari «avec de bonnes intentions» aucuns dommages-intérêts exemplaires ne peuvent être demandés» ${ }^{29}$.

$\mathrm{Si}$ on est convaincu du fait que les gouvernements islamistes venant au pouvoir après le printemps arabes continueront à mettre en place les mesures nécessaires pour le processus démocratique, il reste à craindre le comment. Autrement comment conçoiventils cette notion? Quelle est la conception de la démocratie selon eux? Cette interrogation est légitime en considérant que le lendemain des révolutions arabes les islamistes venant au pouvoir ont refusé les quotas ce qui a affecté la participation politique des femmes. En Egypte par exemple, le parti salafiste égyptien Al- Nour a mis une fleur à la place de la photo de chaque femme élue, de plus les femmes membres du parlement ne doivent être ni vues, ni entendues ${ }^{30}$. Quelle démocratie celle qui interdit aux femmes de faire entendre leur voie ? La démocratie est conditionnée entre autres par la liberté dont le respect des droits humaines constitue un élément fondamental comme le confirme le préambule de la déclaration universelle des droits de l'Homme de 1948 "la reconnaissance de la dignité inhérente à tous les membres de la famille humaine et de leurs droits égaux et inaliénables constitue le fondement de la liberté, de la justice et de la paix dans le monde» ${ }^{31}$. Il reste à dire que la question des droits des femmes constitue le vrai thermomètre de la compatibilité entre l'islam politique et la démocratie.

\footnotetext{
27 Chef du Gouvernement, Programme gouvernemental, Rabat, www.protailmaroc.ma

28 Ricardo Hausmann, Laura D. Tyson, Saadia Zahidi, The Global Gender Gap Report 2012 Ranking and Scores, World Economic Forum, 2012, http://www3.weforum.org/docs/WEF GenderGap Report 2012.pdf

29 Mona Eltawahy, Why do hate us? The real war on women is in the Middle East, Foreign policy magazine, May/June 2012. http://www.foreignpolicy.com/articles/2012/04/23/why_do_they_hate_us?page =full

30 Ibid.

31 Déclaration Universelle des Droits de l'Homme de 1948.
} 
La démocratie ne peut avoir de signification véritable qu'à travers des mesures concrètes en faveur de la parité et l'instauration des droits des femmes dans leur totalité et sans réserve dans tous les domaines : santé, enseignement, emploi, lutte contre la violence à l'encontre des femmes, etc. Pourtant les femmes sont marginalisées dans tous les pays arabes. Au Maroc, par exemple, le gouvernement à écarter le mouvement féminin qui a milité pour l'intégration du principe de la parité et de l'autorité pour la lutte contre la discrimination dans la nouvelle constitution.

Le planning législatif du gouvernement qui vise l'activation de l'application de la constitution de 2011 stipule l'investiture de commissions indépendantes pour la création des trois instances de protection des droits humains, affecte la mission de créer l'instance de la parité et de la lutte contre toutes les formes de discrimination, au ministère de la Solidarité, de la Femme, de la Famille et du développement social ${ }^{32}$.

Ce Ministère prépare l'investiture d'une commission dite "scientifique" chargée du suivi de la préparation d'un projet de loi portant création de l'instance de la parité et de la lutte contre toutes les formes de discrimination sans aucune consultation des associations féminines. Cet acte traduit le non respect des nouvelles dispositions de la nouvelle constitution principalement les articles 12, 13 et 14 en ce qui concerne la participation de la société civile au travail de l'exécutif et du législatif et le non respect de l'article 19 à propos de la parité et de la lutte contre toutes les formes de discrimination ${ }^{33}$.

En définitive, certes le printemps arabe a été marqué par un déclin en ce qui concerne les droits des femmes, pourtant il a donné lieu à la naissance de plusieurs réseaux féministes et il a donné un autre souffle aux mouvements féminins qui ont commencé à s'organiser et à renforcer leurs compétences de plaidoiries tout en utilisant les medias sociaux. Cela ne peut que nous rendre plus optimiste pour dire que ces femmes qui ont initiées les révolutions arabes sont aptes à mener une autre révolution pour préserver leur droits et arracher d'autres. Une nouvelle révolution se prépare, une révolution des femmes arabes pour une réelle démocratie.

\footnotetext{
32 Le printemps Féminin pour l'Egalité et la Démocratie, A qui revient la responsabilité de superviser la création de l'instance de la parité et de la lutte contre toutes les formes de discrimination ?, Communiqué de presse, Casablanca, le 09/02/2013. www.flddf.ma

33 Ibid.
} 


\title{
RÉFÉRENCES BIBLIOGRAPHIQUES
}

\author{
Chef du Gouvernement, Programme gouvernemental, Rabat, 2011 www.protailmaroc.ma
}

Conseil de l'Europe, Egalité entre les femmes et les hommes : une condition du succès du printemps arabe, Assemblée Parlementaire, 05 avril 2012, http://assembly.coe.int

Armogathe, D. (1977) Le deuxième sexe Beauvoir. Paris: Hatier.

Déclaration Universelle des Droits de l'Homme de 1948.

Truchet, D. (2010) Le droit public. Paris: PUF. Fédération internationale des ligues des droits de l'Homme, Monde arabe : quel printemps pour les femmes, document électronique, (2012) www. fidh.org

Dupuis-Déri, F. (1994) Qu'est-ce que la démocratie?, Horizons philosophiques, vol. 5, $\mathrm{n}^{\circ} 1$, disponible en Internet http://id.erudit.org/iderudit/800967ar.

Françoise Nduwimana, La Résolution 1325 du Conseil de sécurité de l'ONU sur les femmes, la paix et la sécurité Comprendre les implications, remplir les obligations, Bureau de la Conseillère spéciale pour la parité entre les sexes et la promotion de la femme OSAGI Département des Affaires économiques et sociales (DAES), Commission économique des Nations Unies pour l'Afrique (CEA) New York. Document d'internet site web : http://www.un.org/womenwatch/osagi/cdrom/documents/Background_Paper_Africa_fr.pdf.

Haut Commissariat au Plan, (2009) Enquête nationale sur la prévalence de la violence à l'égard des femmes au Maroc, document d'internet site web : http://www.hcp.ma

Le Printemps Féminin pour l'Egalité et la Démocratie, A qui revient la responsabilité de superviser la création de l'instance de la parité et de la lutte contre toutes les formes de discrimination?, Communiqué de presse, Casablanca, le 09/02/2013. Document d'internet site web http:// www.flddf.ma.

Jean-Pierre PUISSOCHET, Hubert LEGAL, Le principe de sécurité juridique dans la jurisprudence de la Cour de justice des Communautés européennes, Cahiers du Conseil constitutionnel $n^{\circ} 11$ (Dossier: Le principe de sécurité juridique), décembre 2001 p7, document d'internet site web http://www.conseil-constitutionnel.fr/conseil-constitutionnel/francais/cahiers-du.

Karim Hussein, Donata Gnisci et Julia Wanjiru, Sécurité et sécurité humaine: Présentation des concepts et des initiatives, Quelle conséquences pour l'Afrique de l'Est, OCDE, 2004, p12. Document d'internet site web http://www.oecd.org/fr/csao/publications/38826711.pdf

Eltawahy, M. (Mayo - Junio 2013) Why do hate us? The real war on women is in the Middle East, Foreign policy magazine, document d'internet site web: http:/www.foreignpolicy.com/ articles/2012/04/23/why_do_they_hate_us?page=full.

Banerjee, P., Poutiainen, P., Dey, I., Dunghana, S., Kiolo, w., Matila, P. et Muhindi, M. (2010) Women, Peace and Security, Implementation of UN Security Council Resolution 1325 in the context of Finnish Development Policy with case studies from Kenya, Nepal and North-Est India, Finnish Ministry for Foreign Affairs, Helsinki. 
Programme des Nations Unies de Développement, Les défis de la sécurité humaine dans les pays, Rapport sur le développement humain dans le monde Arabe 2009, p. 22. Document d'internet site web http://www.arab-hdr.org/publications/other/ahdr/ahdr2009f.pdf

Programme des Nations Unies de Développement, L'essor du Sud, le progrès humain dans un monde diversifié, Rapport sur le développement humain 2013, Document d'internet site web http:// www.undp.org/content/dam/undp/library/corporate/HDR/2013GlobalHDR/French/HDR2013\%20 Report\%20French.pdf

Hausmann, R., Tyson, L. D., Zahidi, S. (2012) The Global Gender Gap Report, Ranking and Scores, World Economic Forum, 2012, Document d'internet site web http://www3.weforum.org/ docs/WEF_GenderGap_Report_2012.pdf 